\title{
Tracking the role of anammox in microbial nitrogen cycling using nitrogen and oxygen isotopic measurements
}

\author{
PAUL M MAGYAR ${ }^{1}$, ROBERT NIEDERDORFER ${ }^{2}$, \\ DAMIAN HAUSHERR ${ }^{2}$, HELMUT BÜRGMANN ${ }^{2}$, \\ ADRIANO JOSS $^{2}$, JOACHIM MOHN ${ }^{3}$ AND MORITZ F. \\ LEHMANN $^{1}$ \\ ${ }^{1}$ University of Basel \\ ${ }^{2}$ Eawag, Swiss Federal Institute of Aquatic Science and \\ Technology \\ ${ }^{3}$ Empa, Swiss Federal Laboratories for Materials Testing and \\ Research \\ Presenting Author: paul.magyar@unibas.ch
}

Anammox is a central process in the biological nitrogen cycle that facilitates the anaerobic conversion of nitrite and ammonium into $\mathrm{N}_{2}$ and nitrate. It plays an important role as one of two known processes that removes fixed nitrogen from the environment, and by facilitating the anaerobic conversion of nitrite and ammonium into $\mathrm{N}_{2}$ and nitrate, functions as a selfcontained nitrogen conversion network. There is also emerging evidence of variations in anammox metabolism and physiology that make it yet more complex.

We have performed batch stable isotope fractionation experiments in wastewater treatment systems performing anammox. These systems feature complex biofilms retained on plastic carriers that are composed of $\sim 10 \%$ anammox bacteria, but whose activity is dominated by the anammox metabolism, as shown by metagenomic, metatranscriptomic, and stoichiometric analyses. These systems can be used to draw connections between the study of anammox in pure or enriched culture-based experiments and more complex natural or engineered settings. We find that the nitrogen isotope effect imparted by ammonium consumption, ${ }^{15} \varepsilon\left(\mathrm{NH}_{4}^{+}\right)$, varies broadly, between $\sim 15 \%$ and $30 \%$, and appears to be controlled by the supply of ammonium. The isotope effect associated with the conversion of nitrite to $\mathrm{N}_{2}$, ${ }^{15} \varepsilon\left(\mathrm{NO}_{2}^{-}-\mathrm{N}_{2}\right)$, appears to be relatively stable at $13.5 \% \pm 3.7 \%$, matching the stability seen in microbial community data. Together this supports the conclusion from past pure culture studies that this parameter reflects what anammox species are present. Finally, in a two-month temperature-perturbation and response experiment we found that nitrogen and oxygen isotope measurements in nitrite and nitrate reflected shifts observed in microbial community and chemical data, and therefore can be used to complement and extend these other approaches, and to diagnose microbial processes in a complex system. 